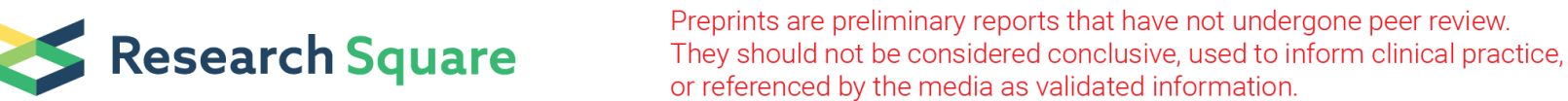

\section{Variants Around miR-451 Can Affect the Risk of Large-artery Atherosclerosis Stroke in Chinese}

Jinyu Gu

Jinling clinical college of nanjing medical university https://orcid.org/0000-0002-4528-3433

Wuzhuang Tang

the affiliated yixing hospital of jiangsu university and yangzhou university

\section{Chong Shen}

Nanjing Medical University

\section{Zibao Li}

Yijishan Hospital of Wannan Medical College

Jie Li

yixing hospital of jiangsu university and yangzhou university

\section{Zhanyun Ren}

yixing hospital of jiangsu university and yangzhou university

\section{Feng Chen}

yixing hospital of jiangsu university and yangzhou university

\section{Huihua Zong}

yixing hospital of jiangsu university and yangzhou university

\section{Chao Wang}

yixing hospital of jiangsu university and yangzhou university

\section{Wenchao Chen}

yixing hospital of jiangsu university and yangzhou university

\section{Yunzi Li}

jinling hospital of nanjing university

Yun Li

jinling hospital of nanjing university

\section{Zhizhong Zhang}

jinling hospital of nanjing medical university and nanjing university

Xinfeng Liu ( $\nabla$ xfliu2@vip.163.com )

Jinling clinical college of nanjing medical university and nanjing university

\section{Research Article}

Keywords: microRNA, polymorphism, ischemic stroke, intergenic region, rs901975 
Posted Date: April 28th, 2021

DOl: https://doi.org/10.21203/rs.3.rs-450391/v1

License: (c) (i) This work is licensed under a Creative Commons Attribution 4.0 International License. Read Full License 


\section{Abstract}

Background: Ischemic stroke has high incidence rate, mortality rate and disability rate. Genetic factors have a significant impact on stroke risk. MicroRNAs are a class of small non-coding RNA. Intergenic variants can affect the regulation of microRNAs and modulate large-artery atherosclerosis stroke susceptibility. The low expression of miR-451 aggravated ischemic injury significantly.

Methods: Functional intergenic variants near hsa-mir-451 were identified by bioinformatics analysis. We conducted a case-control study to explore the associations of selected variants with large-artery atherosclerosis stroke risk in Chinese.

Results: The rs901975 (G>A) near hsa-mir-451 was identified as a functional SNP for stroke susceptibility. The protective effect of $A$ allele was significant in codominant model $(O R=0.62,95 \% \mathrm{Cl}=$ 0.44-0.89, $P=0.005)$, recessive model $(\mathrm{OR}=0.65,95 \% \mathrm{Cl}=0.47-0.89, P=0.006)$ and log-additive model $(\mathrm{OR}=0.82,95 \% \mathrm{Cl}=0.71-0.96, P=0.012)$. We also found the significant effect in participants over 65 years old, male, hypertensive and diabetic people. Moreover, hypertensive people genotyped as GG+GA had 2.84 - fold increased risk compared with those genotyped as AA without hypertension $\left(P_{\text {interaction }}=\right.$ 0.036). In the MEGASTROKE Consortium, rs901975 A allele also had protective effect on LAA stroke in Europeans (OR $=0.937,95 \% \mathrm{Cl}: 0.882-0.996, P=0.036)$. Combined analysis of our study and MEGASTROKE showed consistent trend (OR $=0.920,95 \% \mathrm{Cl}: 0.869-0.973, P=0.004)$.

Conclusions: Our study suggested that rs901975 near hsa-mir-451 might affect large-artery atherosclerosis stroke susceptibility in Chinese population.

\section{Introduction}

Stroke is going to be an epidemic worldwide. The high disability rate and mortality of stroke is a challenge for health (Hankey 2017). About $80 \%$ of stroke are ischemic stroke (IS) (Benjamin et al. 2019). IS was classified to be five etiological subtypes according to the TOAST criteria (Adams et al. 1993). Among those subtypes, large-artery atherosclerosis (LAA) stroke is more prevalent in Chinese population (Liu et al. 2011). Clinical trials have shown that hypertension, hypercholesterolemia, and carotid stenosis are causal risk factors for LAA stroke (Hankey 2017). But these factors cannot explain all the causes of stroke. Studies in family found the importance of genetic risk factors involving in the progress of stroke (Fan et al. 2019). The role of single nucleotide polymorphisms (SNPs) in interleukin 6 (Akinyemi et al. 2017), paraoxonase 1 (Wei et al. 2017), C-reactive protein (Ye et al. 2018) and so on has been confirmed to be responsible for stroke susceptibility. The discovery of $A N R / L$ prove the function of non-coding RNA (ncRNA) on stroke (Pasmant et al. 2011).

MicroRNAs (miRNAs) are a class of ncRNA with $\sim 23$ nucleotides. They play a negative regulatory mechanism by post transformation modification regulation. It mainly combines with 3 ' untranslated region (UTR) of mRNA to induce mRNA degradation. When only part of sequences is paired, it can inhibit mRNA translation (Bartel 2009). Atherosclerosis is mainly caused by lipid metabolism disorder and leads 
to functional changes of endothelial cells, smooth muscle cells, and leukocyte recruitment (Weber and Noels 2011). A series of studies revealed the importance of miRNAs such as miR-181b, miR-146a, miR$143 / 145$ and so on in molecular signaling pathways and lipid homeostasis involved in atherosclerosis (Feinberg and Moore 2016). The miR-451 may suppress the inflammatory response mediated by microglia activation, targeting TLR4 (Toll-like receptor 4), but the overexpression of miR-451 offsets the inhibitory effect (Sun and Zhang 2018). miR-451 has been detected as biomarker and potential regulation target in tumors (Bai and Wu 2019), rheumatoid arthritis (Churov et al. 2015) and amyotrophic lateral sclerosis (Dolz et al. 2017). Moreover, the association between stroke and miR-451 is also being investigated in vivo and in vitro. In a Chinese group, miR-451 in circulation showed a lower level in patients compared with the controls and in a mouse middle cerebral artery occlusion (MCAO) model, upregulation of miR-451 can reduce ischemia-reperfusion injury (Fu et al. 2019).

Many disease-associated SNPs (daSNPs) have been indicated by Genome-wide association studies (GWAS). SNPs located in mature miRNAs or pre-miRNAs could cause complex influence in miRNAs processing, expression, and/or binding to target mRNA (Hu et al. 2008). However, only parts of SNPs are in the functional genes. The daSNPs in intergenic regions (IGR) are also remarkable in disease phenotypes. The IGR-daSNPs can regulate target genes by disrupt the function of nearby host regulatory elements (HREs), causing abnormal expression of disease related genes, and consequently leading to disease. (Chen and Tian 2016).

A series of studies have reported SNPs in miRNAs can affect stroke susceptibility. The relationship of SNPs in IGR of pre-miRNAs on LAA stroke is still unclear. Thus, we tried to identify the functional SNPs ground IGR of the hsa-mir-451 and evaluated their effect on the LAA stroke risk in Chinese.

\section{Methods}

\subsection{Subjects}

The diagnosis of LAA stroke is mainly based on the medical history, focal neurological deficit and imaging findings of intracranial and extracranial vascular stenosis over $50 \%$. All individuals in this study have been described previously (Yang et al. 2018). In short, 746 patients with LAA stroke and 1,101 controls were finally included in this study. All patients were initially diagnosed as LAA stroke according to TOSAT by experienced clinician at enrollment. Those who are younger than 18 , have cancer, autoimmune diseases or organ failure were excluded. The 1,101 controls were selected from healthy individuals without a history of cardiovascular or cerebrovascular disease during the same period. This study was approved by an ethical review in Jinling Hospital, Nanjing, China and each participant gave a written informed consent.

\subsection{SNP selection and genotyping}

Based on the UCSC Genome Browser database (http://genome.ucsc.edu/, 2020-03-05), we browsed $5 \mathrm{~kb}$ upstream and $5 \mathrm{~kb}$ downstream IGR of hsa-mir-451. The 310bp 3' of hsa-mir-451 is the coding region of 
ERAL 1. Thus, we only chose the $5 \mathrm{~kb} 5$ ' flanking region of hsa-mir-451 and 13 SNPs was found in Chinese Han South population (Supplementary Table 1). After removing the SNPs with more than two alleles, 4 common SNPs with minor allele frequencies (MAF) $\geq 5 \%$ were selected. Then we used rSNPBase (http://rsnp.psych.ac.cn/, 2020-03-05) to find potentially functional SNPs that might be an eQTL of genes or have association with regulatory elements. Finally, rs901975 was included in our study.

The genotyping methods has been described before (Gu et al. 2021). Finally, 715 patients with LAA stroke and 1,088 controls were genotyped successfully. The call rate was $97.6 \%(1,803 / 1,847)$.

\subsection{Statistical Analysis}

We used Student's $t$ test and chi-squared test to compare continuous and categorical variables respectively. Hardy-Weinberg equilibrium (HWE) of allele frequencies was tested by $c^{2}$ test in control group. Logistics regression was used to test the association of rs901975 with LAA stroke susceptibility in log-additive, codominant, codominant, and recessive models. Crude and adjusted odds ratios (ORs) and 95\% confidence intervals (Cls) were calculated to evaluate the effect of genetic model. The results of stratified analysis were shown as forest plot. We tried to find the interaction between SNP and other factors. Two-tailed $P<0.05$ ( $\mathrm{a}=0.05)$ was considered significant. R software (version 3.5.1) was used for all the statistical analyses.

\section{Results}

\subsection{Individual characteristics}

Characteristics of individuals have been described in Table 1. There was no significant difference in age $(P=0.450)$ and $\operatorname{sex}(P=0.167)$ distribution between cases and controls. The patients have a higher proportion of hypertension and diabetes compared with the controls $(P<0.001)$. 
Table 1

Baseline information.

\begin{tabular}{|llll|}
\hline Variables & Cases $\mathbf{N = 7 4 6}$ & Controls $N=1101$ & $P$ \\
\hline Age (y, SD) & n (\%) & $\mathrm{n}(\%)$ & \\
\hline Sex & & & \\
\hline Male & $459(61.5)$ & $642(58.3)$ & 0.1650 \\
\hline Female & $287(38.5)$ & $459(41.7)$ & \\
\hline Hypertension & & & \\
\hline No & $203(27.2)$ & $599(54.4)$ & $<0.001$ \\
\hline Yes & $543(72.8)$ & $502(45.6)$ & \\
\hline Diabetes & & & $<0.001$ \\
\hline No & $556(74.5)$ & $1065(96.7)$ & \\
\hline Yes & $190(25.5)$ & $36(3.3)$ & \\
\hline
\end{tabular}

\subsection{Association between SNP rs901975 and LAA stroke risk}

The MAF of rs 901975 were 0.34 in cases and 0.38 in controls, respectively among the successfully genotyped people. The distribution of allele frequency for the control group conformed to HWE $(P=$ 0.460). The AA genotype of rs 901975 was associated with a $38 \%$ decreased risk on LAA stroke compared with the GG genotype in codominant model (adjusted for age, sex, hypertension, and diabetes, $95 \% \mathrm{Cl}=$ $0.44-0.89, P=0.005)$. The protective effect of A allele is still significant in other genetic models (recessive model AA vs GG + GA: AOR $=0.65,95 \% \mathrm{Cl}=0.47-0.89, P=0.006$; log-additive model: $\mathrm{AOR}=$ $0.82,95 \% \mathrm{Cl}=0.71-0.96, P=0.012 ;$ Table 2). 
Table 2

Association between rs901975 and LAA stroke.

\begin{tabular}{|lllllll|}
\hline Genotypes & & Case/Control & COR $(95 \% \mathrm{Cl})$ & $\boldsymbol{P}$ & AOR $(95 \% \mathrm{Cl})$ & $\boldsymbol{P}^{*}$ \\
\hline Codominant & GG & $310 / 423$ & 1 & 1 & \\
& AG & $329 / 505$ & $0.89(0.73-1.09)$ & 0.253 & $0.92(0.74-1.15)$ & 0.450 \\
\hline & AA & $76 / 160$ & $0.65(0.48-0.88)$ & 0.006 & $0.62(0.44-0.89)$ & 0.005 \\
\hline Dominant & G/G & $310 / 423$ & 1 & 1 & \\
\hline Recessive & G/G + G/A & $405 / 665$ & $0.83(0.69-1.01)$ & 0.058 & $0.84(0.69-1.04)$ & 0.108 \\
\hline & A/A & $769 / 928$ & 1 & & 1 & \\
\hline Log-additive & & & $0.69(0.52-0.92)$ & 0.011 & $0.65(0.47-0.89)$ & 0.006 \\
\hline CoR: crude odd ratio; AOR: adjusted odd ratio; *adjusted by age, sex, hypertension, and diabetes. \\
\hline
\end{tabular}

\subsection{Stratified analysis}

Furthermore, stratified analysis was performed under the significant codominant and recessive genetic models of rs901975 (Fig. 1). For those who are older than 65 years, the protective effect of rs 901975 AA was prominent both in codominant and recessive model (AA vs GG: AOR $=0.51,95 \% \mathrm{Cl}=0.32-0.81, P=$ $0.005 ; A A$ vs $\mathrm{GG}+\mathrm{AG}: \mathrm{AOR}=0.55,95 \% \mathrm{Cl}=0.35-0.86, P=0.008)$. In male individuals, the rs $901975 \mathrm{AA}$ played significant protective effect on LAA stroke (AA vs GG: AOR $=0.58,95 \% \mathrm{Cl}=0.38-0.89, P=0.012$; AA vs $G G+A G: A O R=0.61,95 \% \mathrm{Cl}=0.41-0.91, P=0.014)$. The rs901975 AA decreased the LAA stroke risk in hypertensive people (AA vs GG: AOR $=0.49,95 \% \mathrm{Cl}=0.32-0.75, P=0.001 ; \mathrm{AA}$ vs $\mathrm{GG}+\mathrm{AG}$ : $\mathrm{AOR}=$ $0.51,95 \% \mathrm{Cl}=0.35-0.76, P=0.001)$. The protective effect was still significant in non-diabetic population (AA vs GG: $A O R=0.56,95 \% \mathrm{Cl}=0.39-0.80, P=0.002 ; \mathrm{AA}$ vs $\mathrm{GG}+\mathrm{AG}$ : $\mathrm{AOR}=0.61,95 \% \mathrm{Cl}=0.44-0.86, P=$ $0.004)$. No significant protective effect of $A$ allele was observed among the subjects aged $\leq 65$ years, female, without hypertension and with diabetes under the two genetic model.

\subsection{Interaction between hypertension and rs901975}

Hypertension is also a manifestation of atherosclerosis. In people with hypertension, the LAA stroke risk caused by rs 901975 were still significant. Interaction analysis found an additional increased risk of LAA stroke (adjusted by age, sex and diabetes, Fig. 2). Compared to the non-hypertensive people with rs901975 AA, the hypertensive people with rs $901975 \mathrm{GG}$ had 2.97 - fold increased risk (OR $=2.97,95 \% \mathrm{Cl}$ : $\left.1.77-4.98, P=3.5 \times 10^{-5}\right)$. There was no significant multiplicative interaction under codominant model $(P$ $=0.110)$. Under the recessive genetic model, rs $901975 \mathrm{GG}+\mathrm{GA}$ genotype with hypertension had 2.84 fold increased risk compared with AA genotype without hypertension $(\mathrm{OR}=2.84,95 \% \mathrm{Cl}: 1.74-4.65, P=$ $\left.3.2 \times 10^{-5}\right)$. The multiplicative interaction was still significant $(P=0.036$, Table 3$)$. 
Table 3

The interaction between rs901975 and hypertension on LAA stroke.

\begin{tabular}{|llllll|}
\hline rs901975 & hypertension & Case/control & AOR $(95 \%$ Cl $)$ & $P$ & Interaction $P$ \\
\hline AA & no & $25 / 72$ & 1 & & 0.110 \\
\hline AA & yes & $51 / 88$ & $1.45(0.80-2.63)$ & 0.224 & \\
\hline AG & no & $86 / 277$ & $0.95(0.55-1.61)$ & 0.834 & \\
\hline AG & yes & $243 / 228$ & $2.74(1.65-4.55)$ & $1.04 \times 10^{-4}$ & \\
\hline GG & no & $84 / 242$ & $1.04(0.61-1.78)$ & 0.890 & \\
\hline GG & yes & $226 / 181$ & $2.97(1.77-4.98)$ & $3.50 \times 10^{-5}$ & \\
\hline AA & no & $25 / 72$ & 1 & & 0.036 \\
\hline AA & yes & $51 / 88$ & $1.45(0.78-2.63)$ & 0.224 & \\
\hline AG + GG & no & $170 / 519$ & $0.99(0.60-1.64)$ & 0.964 & \\
\hline AG + GG & yes & $469 / 409$ & $2.84(1.74-4.65)$ & $3.20 \times 10^{-5}$ & \\
\hline AOR: adjusted odd ratio by age, sex, hypertension, and diabetes. & & \\
\hline
\end{tabular}

\subsection{Bioinformatics information}

The allele frequencies of rs901975 for different races were explored on Emsembl website (http://asia.ensembl.org/, 2020-03-05, Supplementary Table 2). The minor allele of the SNP rs901975 was $A$ in all races. Based on HaploReg v4.1

(http://pubs.broadinstitute.org/mammals/haploreg/haploreg.php/, 2020-03-16), SNP rs901975 and its nearby variants within a LD block are located in regulatory regions, and to be an eQTL for genes in brain and blood. The potential function of rs 901975 and has-miR-451 were showed in Fig. 3. On miRWalk 3.0 web tool (http://mirwalk.umm.uni-heidelberg.de/, 2020-03-16), we browsed target genes of hsa-miR-451 and found that VANGL2, MCTS1 and POM121C are mentioned in both TargetScan and miRDB database in Human. The rs901975 is in promoter and enhancer sites. This SNP is an eQTL for DHRS13 in brain and cerebellar hemisphere $\left(P=6.65 \times 10^{-6}\right)$. These evidences indicate that rs 901975 is related to the functional regulation of genes in brain. Analyses in the MEGASTROKE (Malik et al. 2018) yielded consistent trends for LAA stroke $(\mathrm{OR}=0.937,95 \% \mathrm{Cl}$ : $0.882-0.996)$. Combined analysis of our study and MEGASTROKE showed that rs901975 A allele was associated with decreased risk of LAA stroke (OR = $0.920,95 \% \mathrm{Cl}: 0.869-0.973, P=0.004)$.

\section{Discussion}

Numerous predisposing risk factors involved in LAA stroke. Genetic factors can explain the risk of stroke which cannot be covered by traditional risk factors. Heritability for LAA stroke was described as $40.3 \%$ 
(Bevan et al. 2012). The genetic risk factors play significant roles in the diagnosis and prevention of LAA stroke. MiRNAs are a group of ncRNA with about 23 nucleotide length. They can regulate gene expression by targeting the 3'UTR of the mRNA. Growing evidence has indicated circulating miRNAs can be unique biomarkers for stroke diagnosis, prognosis, and therapy (Mirzaei et al. 2018). In previous studies, our team found that microRNA related SNPs can affect LAA stroke susceptibility (Wang et al. 2021).

The associations of SNPs in $5 \mathrm{~kb} 5$ flanking region of hsa-mir-451 with the LAA stroke risk were explored in our study. Our findings suggested that rs901975 (G >A) near hsa-mir-451 was associated with a decreased LAA stroke risk in Chinese population. The results of stratified analysis based on age, sex, hypertension, and diabetes for codominant and recessive genetic model of rs901975 were also significant. The protective effect of rs901975 A allele was also confirmed in other ethnic groups in MEGASTROKE.

In a research for human myocardial infarction (MI), miR-451 levels were up-regulated in 7-day MI (Bostjancic et al. 2009). The miR-451 is a special miRNA because pri-miR-451 is too short to be recognized by Dicer and it requires Argonaute2 catalytic activity (Cifuentes et al. 2010). Researchers often pay attention to miR-451 when they study miRNA function. miR-451 targets CUGBP Elav-like family member 2, which protects neurons from apoptosis and oxidative stress induced by oxygen and sugar deprivation/reoxygenation (Liu et al. 2018). By targeting TLR4, miR-451 could inhibit microglia activationmediated inflammation, which is common in the injury and reparation of brain tissue in stroke (Sun and Zhang 2018). The miR-451 has protective effect on cerebral ischemia-reperfusion injury both in mice and human. It was speculated that higher miR-451 levels relate to a good prognosis for stroke patients. (Fu et al. 2019).

The rs 9019751 is a common $\mathrm{G}>\mathrm{A}$ variant on chromosome $17,1 \mathrm{~kb} 5^{\prime}$ of $m i R-451$. Unfortunately, there was no evidence that the miR-451 directly match rs901975 in the existing databases. SNP rs9019751 is in the intergenic region, between genes. Intergenic genomic sequences have been considered as desert DNA fragments in the past time. But it is currently known that approximately $98 \%$ of intergenic DNA sequences are related to ncRNA (Lander et al. 2001). Intergenic variants may cause disease through regulation of ncRNA expression. It has been found that some intergenic SNPs, such as rs12425791 within $11 \mathrm{~kb}$ of NINJ2 (Nie et al. 2019), can affect stroke susceptibility.

To date, no studies had shown the exact mechanism of the rs901975 regulating the function of miR-451 or other mRNAs. Parts of the associations between the rs 901975 and clinical features have been researched. The rs901975 was found to associate with low-density lipoprotein (LDL) in a summary databased Mendelian randomization (Pavlides et al. 2016). Thus, the rs 901975 variant may participate in the process of lipid metabolism disorders in atherosclerosis. The vascular occlusion caused by atherosclerotic plaque and hemodynamic failure are main pathology induced LAA stroke. Oxidized low density lipoprotein (Ox-LDL) has been considered an important risk factor for vascular calcification and atherosclerosis. Knockdown of TLR4 reduced vascular calcification induced by Ox-LDL through nuclear factor kappa B (NK-KB) (Song et al. 2017). The rs901975 and miR-451 may be connected by TLR4. 
Therefore, we speculated that rs 901975 is associated with high level of Ox-LDL, which can negatively regulate TLR4 level and thus increase the level of circulating miR-451. Although rs901975 cannot directly match miR-451 to regulate its circulating level, it can participate in miR-451 induced stroke through other molecular pathways.

In conclusion, our study indicated that the functional intergenic variants near miR-451 were significantly associated with LAA stroke risk. These findings need further functional studies and large populationbased studies to verify.

\section{Declarations}

\section{Funding}

This study was supported by National Natural Science Foundation of China (Grant Nos. 81771285, 81870946 and 81530038) and the China Key Research and Development Project (2017YFC1307901).

\section{Conflicts of interest}

The authors declare that they have no conflict of interest.

\section{Availability of data and material}

The data that support the findings of this study are available from the corresponding author upon reasonable request.

Code availability Not applicable.

\section{Authors' contributions}

Jinyu Gu: Conceptualization, Methodology, Software, Formal analysis, Writing - Original Draft, Visualization. Zhizhong Zhang: Conceptualization, Methodology, Software, Validation, Writing - Review \& Editing, Supervision, Funding acquisition. Xinfeng Liu: Conceptualization, Supervision. Wuzhuang Tang: Resources, Data Curation. Chong Shen: Methodology, Data Curation. Zibao Li: Methodology, Software. Jie Li: Investigation. Zhanyun Ren: Investigation. Feng Chen: Investigation. Huihua Zong: Investigation. Chao Wang: Investigation. Wenchao Chen: Investigation. Yunzi Li: Project administration, Funding acquisition. Yun Li: Investigation.

\section{Ethics approval}

This study was approved by the Ethical Review Committee of Jinling Hospital, Nanjing, China.

\section{Consent to participate}

Informed consent was obtained from all individual participants included in the study. 
Consent for publication Not applicable.

\section{References}

1. Adams HP, Jr., Bendixen BH, Kappelle LJ, Biller J, Love BB, Gordon DL et al. (1993) Classification of subtype of acute ischemic stroke. Definitions for use in a multicenter clinical trial. TOAST. Trial of Org 10172 in Acute Stroke Treatment Stroke 24:35-41

2. Akinyemi R, Arnett DK, Tiwari HK, Ovbiagele B, Sarfo F, Srinivasasainagendra V et al. (2017) Interleukin-6 (IL-6) rs1800796 and cyclin dependent kinase inhibitor (CDKN2A/CDKN2B) rs2383207 are associated with ischemic stroke in indigenous West African Men J Neurol Sci 379:229-235 doi:10.1016/j.jns.2017.05.046

3. Bai H, Wu S (2019) miR-451: A Novel Biomarker and Potential Therapeutic Target for Cancer OncoTargets and therapy 12:11069-11082 doi:10.2147/ott.s230963

4. Bartel DP (2009) MicroRNAs: target recognition and regulatory functions Cell 136:215-233 doi:10.1016/j.cell.2009.01.002

5. Benjamin EJ, Muntner P, Alonso A, Bittencourt MS, Callaway CW, Carson AP et al. (2019) Heart Disease and Stroke Statistics-2019 Update: A Report From the American Heart Association Circulation 139:e56-e528 doi:10.1161/cir.0000000000000659

6. Bevan S, Traylor M, Adib-Samii P, Malik R, Paul NL, Jackson C et al. (2012) Genetic heritability of ischemic stroke and the contribution of previously reported candidate gene and genomewide associations Stroke 43:3161-3167 doi:10.1161/strokeaha.112.665760

7. Bostjancic E, Zidar N, Glavac D (2009) MicroRNA microarray expression profiling in human myocardial infarction Dis Markers 27:255-268 doi:10.3233/DMA-2009-0671

8. Chen J, Tian W (2016) Explaining the disease phenotype of intergenic SNP through predicted long range regulation Nucleic acids research 44:8641-8654 doi:10.1093/nar/gkw519

9. Churov AV, Oleinik EK, Knip M (2015) MicroRNAs in rheumatoid arthritis: altered expression and diagnostic potential Autoimmunity reviews 14:1029-1037 doi:10.1016/j.autrev.2015.07.005

10. Cifuentes D, Xue H, Taylor DW, Patnode H, Mishima Y, Cheloufi S et al. (2010) A novel miRNA processing pathway independent of Dicer requires Argonaute2 catalytic activity Science (New York, NY) 328:1694-1698 doi:10.1126/science.1190809

11. Dolz S, Gorriz D, Tembl JI, Sanchez D, Fortea G, Parkhutik V et al. (2017) Circulating MicroRNAs as Novel Biomarkers of Stenosis Progression in Asymptomatic Carotid Stenosis Stroke 48:10-16 doi:10.1161/STROKEAHA.116.013650

12. Fan M, Lv J, Yu C, Guo Y, Bian Z, Yang S et al. (2019) Family History, Tobacco Smoking, and Risk of Ischemic Stroke J Stroke 21:175-183 doi:10.5853/jos.2018.03566

13. Feinberg MW, Moore KJ (2016) MicroRNA Regulation of Atherosclerosis Circulation research 118:703-720 doi:10.1161/CIRCRESAHA.115.306300 
14. Fu C, Chen S, Cai N, Liu Z, Wang P, Zhao J (2019) Potential Neuroprotective Effect of miR-451 Against Cerebral Ischemia/Reperfusion Injury in Stroke Patients and a Mouse Model World Neurosurg 130:e54-e61 doi:10.1016/j.wneu.2019.05.194

15. Gu J, Shen C, Gu M, Wang M, Zhang Z, Liu X (2021) SNP rs2043211 (p.C10X) in CARD8 Is Associated with Large-Artery Atherosclerosis Stroke in a Chinese Population J Mol Neurosci 71:276283 doi:10.1007/s12031-020-01647-z

16. Hankey GJ (2017) Stroke Lancet (London, England) 389:641-654 doi:10.1016/s01406736(16)30962-x

17. Hu Z, Chen J, Tian T, Zhou X, Gu H, Xu L et al. (2008) Genetic variants of miRNA sequences and nonsmall cell lung cancer survival J Clin Invest 118:2600-2608 doi:10.1172/jci34934

18. Lander ES, Linton LM, Birren B, Nusbaum C, Zody MC, Baldwin J et al. (2001) Initial sequencing and analysis of the human genome Nature 409:860-921 doi:10.1038/35057062

19. Liu L, Wang D, Wong KS, Wang Y (2011) Stroke and stroke care in China: huge burden, significant workload, and a national priority Stroke 42:3651-3654 doi:10.1161/strokeaha.111.635755

20. Liu Q, Hu Y, Zhang M, Yan Y, Yu H, Ge L (2018) microRNA-451 protects neurons against ischemia/reperfusion injury-induced cell death by targeting CELF2 Neuropsychiatr Dis Treat 14:27732782 doi:10.2147/NDT.S173632

21. Malik R, Chauhan G, Traylor M, Sargurupremraj M, Okada Y, Mishra A et al. (2018) Multiancestry genome-wide association study of 520,000 subjects identifies 32 loci associated with stroke and stroke subtypes Nature genetics 50:524-537 doi:10.1038/s41588-018-0058-3

22. Mirzaei H, Momeni F, Saadatpour L, Sahebkar A, Goodarzi M, Masoudifar A et al. (2018) MicroRNA: Relevance to stroke diagnosis, prognosis, and therapy J Cell Physiol 233:856-865 doi:10.1002/jcp.25787

23. Nie F, Yu M, Liu M, Shang M, Zeng F, Liu W (2019) NINJ2 Gene Polymorphisms and Susceptibility to Ischemic Stroke: An Updated Meta-Analysis Current neurovascular research 16:273-287 doi:10.2174/1567202616666190618125241

24. Pasmant E, Sabbagh A, Vidaud M, Bieche I (2011) ANRIL, a long, noncoding RNA, is an unexpected major hotspot in GWAS FASEB J 25:444-448 doi:10.1096/fj.10-172452

25. Pavlides JMW, Zhu Z, Gratten J, McRae AF, Wray NR, Yang J (2016) Predicting gene targets from integrative analyses of summary data from GWAS and eQTL studies for 28 human complex traits Genome Med 8:84-84 doi:10.1186/s13073-016-0338-4

26. Song Y, Hou M, Li Z, Luo C, Ou JS, Yu H et al. (2017) TLR4/NF-kB/Ceramide signaling contributes to Ox-LDL-induced calcification of human vascular smooth muscle cells European journal of pharmacology 794:45-51 doi:10.1016/j.ejphar.2016.11.029

27. Sun $X$, Zhang H (2018) miR-451 elevation relieves inflammatory pain by suppressing microglial activation-evoked inflammatory response via targeting TLR4 Cell Tissue Res 374:487-495 doi:10.1007/s00441-018-2898-7 
28. Wang M, Gu J, Shen C, Tang W, Xing X, Zhang Z et al. (2021) Association of MicroRNA Biogenesis Genes Polymorphisms with Risk of Large Artery Atherosclerosis Stroke Cellular and molecular neurobiology doi:10.1007/s10571-021-01057-8

29. Weber C, Noels H (2011) Atherosclerosis: current pathogenesis and therapeutic options Nature medicine 17:1410-1422 doi:10.1038/nm.2538

30. Wei LK, Au A, Menon S, Griffiths LR, Kooi CW, Irene L et al. (2017) Polymorphisms of MTHFR, eNOS, ACE, AGT, ApoE, PON1, PDE4D, and Ischemic Stroke: Meta-Analysis J Stroke Cerebrovasc Dis 26:2482-2493 doi:10.1016/j.jstrokecerebrovasdis.2017.05.048

31. Yang S, Zhao Y, Tian Y, Chen Y, Zhao X, Li Y et al. (2018) Common variants of ROCKs and the risk of hypertension, and stroke: Two case-control studies and a follow-up study in Chinese Han population Biochimica et biophysica acta Molecular basis of disease 1864:778-783

doi:10.1016/j.bbadis.2017.12.007

32. Ye Z, Zhang H, Sun L, Cai H, Hao Y, Xu Z et al. (2018) GWAS-Supported CRP Gene Polymorphisms and Functional Outcome of Large Artery Atherosclerotic Stroke in Han Chinese Neuromolecular Med 20:225-232 doi:10.1007/s12017-018-8485-y

\section{Figures}




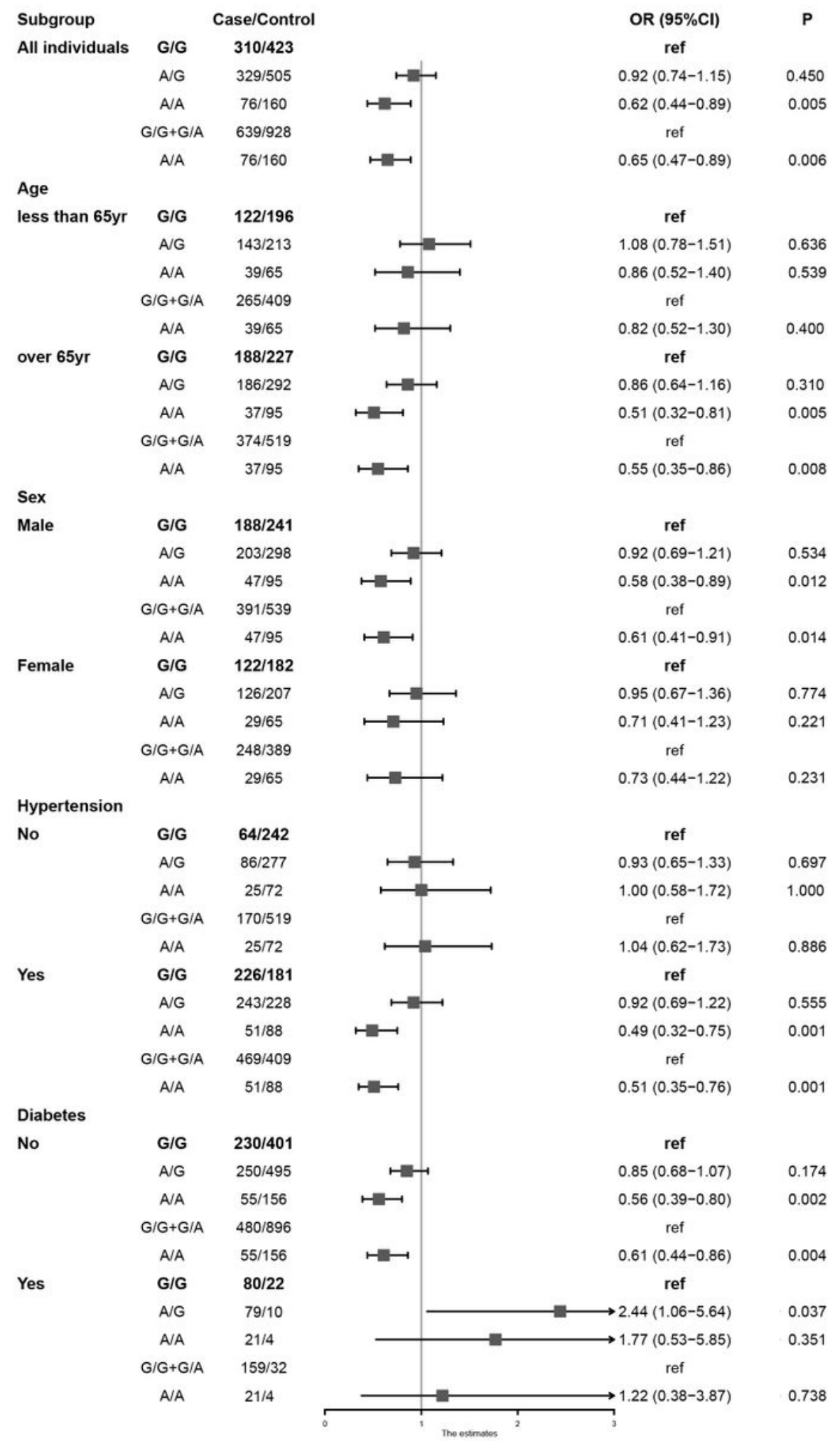

\section{Figure 1}

Forest plot of stratified analysis by age, sex, hypertension, and diabetes for rs 901975 
Fig 2
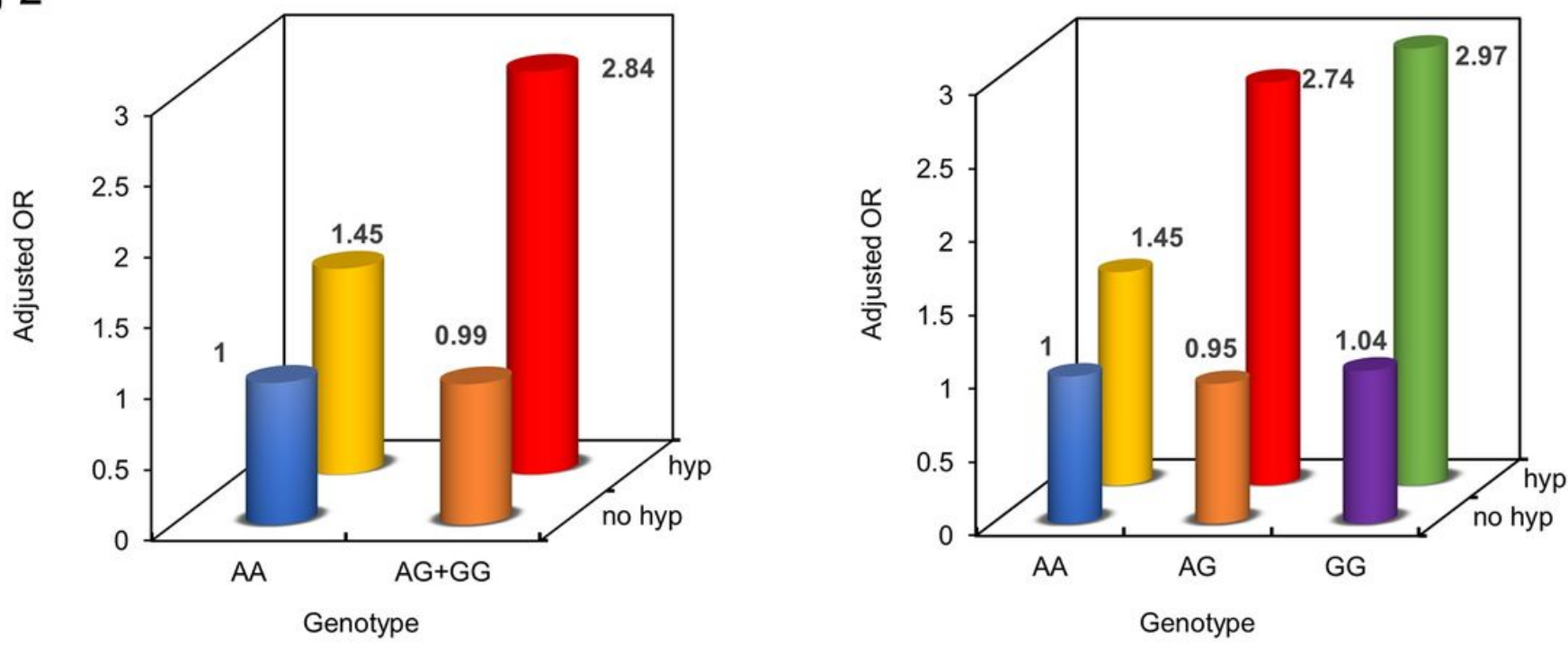

Figure 2

Interaction of rs901975 with hypertension on LAA stroke a. recessive model b. codominant model 

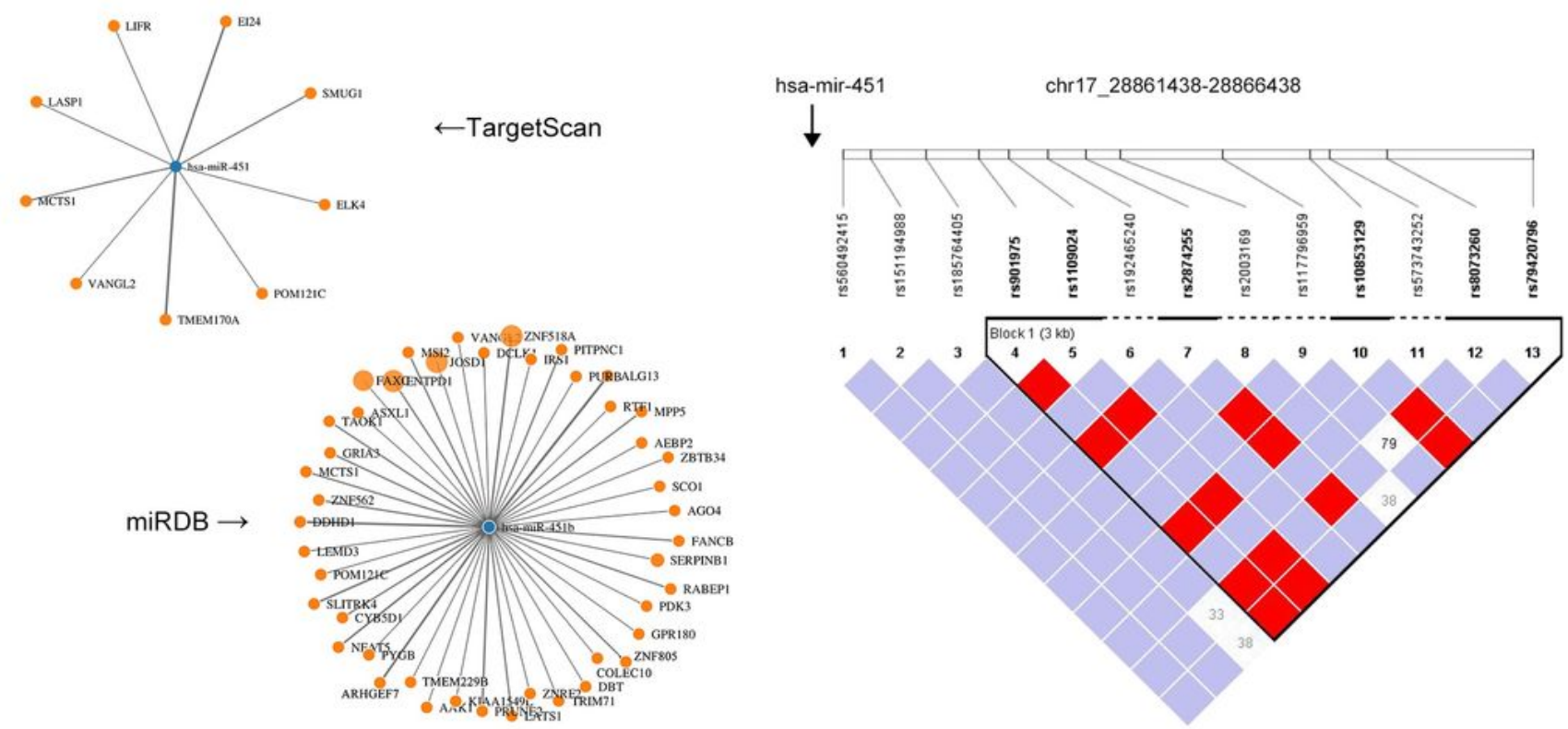

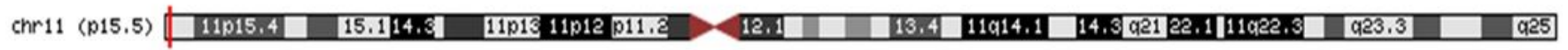

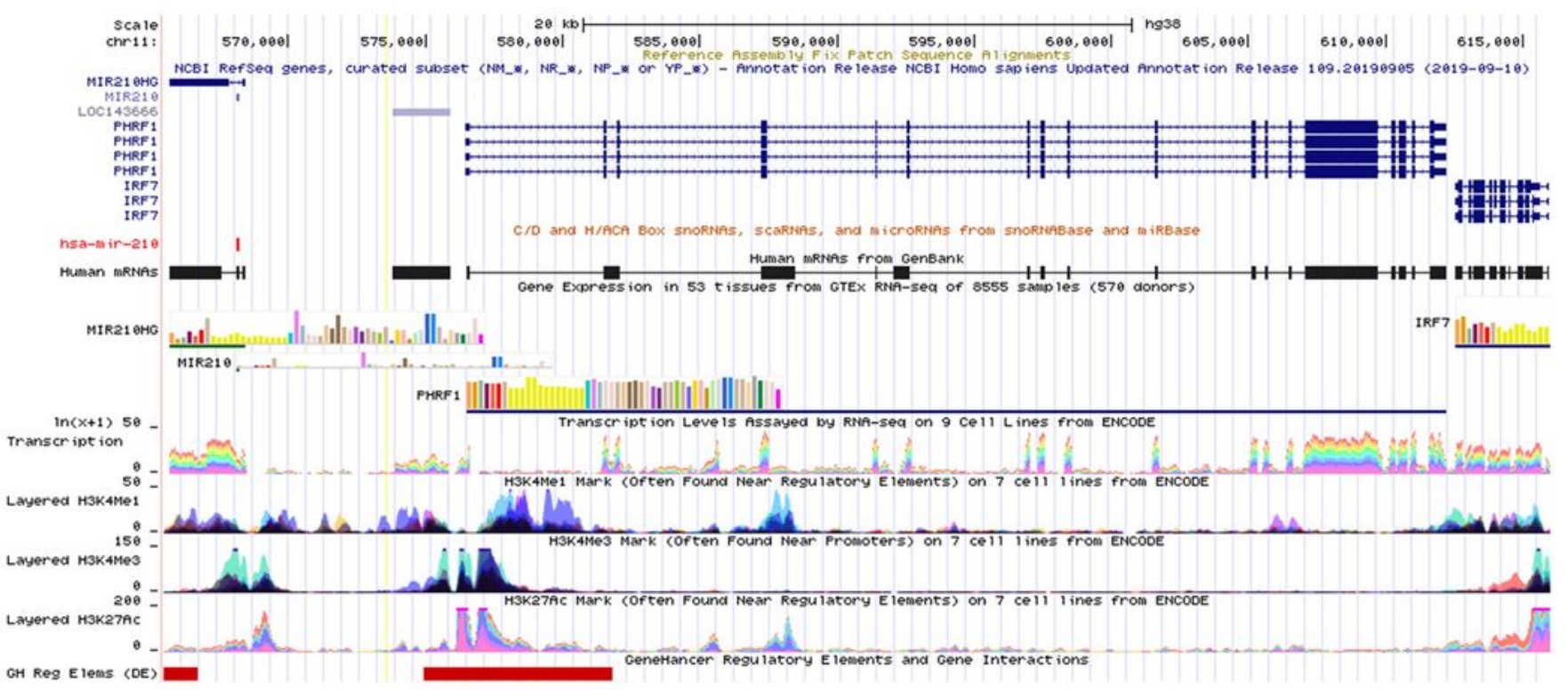

\section{Figure 3}

The potential function of miR-451 and rs901975 (color should be used for this figure in print) a. target genes of hsa-miR-451 based on miRWalk 3.0 b. an LD plot of SNPs in 5kb downstream of hsa-mir-451 c. the figure represents chr17q11.2 within a 20-kb window around rs901975 on UCSC Genome web tool. (NCBI Human Genome GRCh38). Tracks (from top to bottom) in the picture are Genome Base Position, 
Chromosome Bands, NCBI RefSeq Genes, Human mRNAs from GenBank, Transcription Levels Assayed by RNA-seq in 9 Cell Lines from ENCODE and Histone Labeled Functional Sites.

\section{Supplementary Files}

This is a list of supplementary files associated with this preprint. Click to download.

- Supplementarymaterials.docx 